Revue des patrimoines

36 | 2018

Les archives photographiques de presse, pratiques comparées et enjeux méthodologiques

\title{
Les archives photographiques de presse aux Archives nationales : un patrimoine adventice?
}

Newspaper photo archives at the French National Archives, an adventitious heritage?

\section{Sandrine Bula}

\section{(CpenEdition}

\section{Journals}

Édition électronique

URL : http://journals.openedition.org/insitu/17609

DOI : 10.4000/insitu. 17609

ISSN : 1630-7305

Éditeur

Ministère de la culture

\section{Référence électronique}

Sandrine Bula, «Les archives photographiques de presse aux Archives nationales : un patrimoine adventice? », In Situ [En ligne], 36 | 2018, mis en ligne le 15 octobre 2018, consulté le 19 avril 2019. URL : http://journals.openedition.org/insitu/17609 ; DOI : 10.4000/insitu.17609

Ce document a été généré automatiquement le 19 avril 2019

\section{c) $(1) \odot$}

In Situ Revues des patrimoines est mis à disposition selon les termes de la licence Creative Commons Attribution - Pas d'Utilisation Commerciale - Pas de Modification 4.0 International. 


\title{
Les archives photographiques de presse aux Archives nationales : un patrimoine adventice?
}

\author{
Newspaper photo archives at the French National Archives, an adventitious \\ heritage?
}

Sandrine Bula

1 Pourquoi et comment des archives de presse ont-elles rejoint les Archives nationales, institution vouée dès l'origine à recevoir la production des administrations centrales de l'État? La présence de tels fonds se situe au confluent du lent et continu processus de reconnaissance, depuis la fin du $\mathrm{xIx}^{\mathrm{e}}$ siècle, de la valeur historique des sources d'origine privée, et des soubresauts de l'histoire.

2 Deux faits illustrent cette prise de conscience de l'importance des archives autres qu'administratives : en 1930, Lucien Febvre et Charles Schmidt, inspecteur général des bibliothèques et des archives, soulignent dans les Annales l'intérêt fondamental des archives d'entreprises pour la recherche historique ${ }^{1}$. Vingt ans plus tard est créé aux Archives nationales un service dédié aux fonds issus de familles, d'associations et d'entreprises.

3 Par ailleurs, les bouleversements intervenus au lendemain de la Seconde Guerre mondiale dans le secteur de la presse, qui n'avait pas échappé à l'épuration ni à une complète recomposition, conduisirent au sauvetage d'un patrimoine archivistique sur lequel les confiscations de biens et les réaffectations de locaux faisaient planer une menace d'abandon, voire de destruction.

4 Sa préservation fut une préoccupation partagée par le ministère de l'Information, la Bibliothèque nationale, les Archives nationales, ainsi que l'Institut français de presse (IFP), en relation avec l'organisme chargé de gérer à compter de 1946 les biens des entreprises de presse ayant collaboré: la Société nationale des entreprises de presse (SNEP). 
5 Les mesures de sauvegarde visèrent principalement les archives de fonctionnement et la production des organes de presse, mais les fonds photographiques provenant de journaux et d'agences d'illustration de presse furent également pris en compte. Parfois distraits des archives de fonctionnement de l'entreprise, réutilisés par d'autres ou tombés en déshérence, masses encombrantes, peu ou pas identifiées, ils constituaient des objets souvent difficiles à appréhender.

6 Entre les années 1950 et 1990, une douzaine de fonds de presse entrèrent aux Archives nationales, incluant cinq ensembles photographiques de nature et de volumes très variables, allant de «reliquats » de quelques centaines de tirages à des fonds massifs de dizaines de milliers de négatifs sur verre. Restituer l'histoire de leur prise en charge permet de mieux comprendre quel traitement archivistique leur a été appliqué, suivant quelle méthodologie, et comment ils s'apparentent à des fonds similaires conservés dans d'autres institutions patrimoniales.

\section{Le contexte de l'après-guerre : la SNEP}

7 Tous les journaux ayant continué de paraître quinze jours après le début de l'Occupation, soit le 25 juin 1940 pour la zone nord et le 26 novembre 1942 pour la zone sud, furent interdits. Deux ordonnances, du 30 septembre 1944 et du 2 novembre 1945, prévoyaient la mise sous séquestre des biens et actifs ayant servi à la publication des journaux et périodiques visés, en attente d'un jugement ou d'un non-lieu².

8 Le gouvernement attribuait ces biens, moyennant une somme consignée avant l'entrée en possession, aux entreprises de presse nouvellement constituées. Celles-ci devaient verser au propriétaire ou à l'administrateur séquestre une indemnité d'expropriation, déduction faite de la somme consignée. Faute de satisfaire à ces obligations financières, les biens attribués retournaient à l'administration des domaines. Les délais très courts laissés aux nouvelles entreprises les mettaient en difficulté, alors que leur équilibre financier était loin d'être assuré. Ces contraintes, ainsi que les choix d'attribution jugés arbitraires, rendaient les ordonnances difficilement applicables.

9 Une loi, promulguée le 11 mai 1946, devait apporter des solutions, avec la création de la Société nationale des entreprises de presse (SNEP), établissement public à caractère industriel et commercial auquel étaient transférés les biens confisqués. Les entreprises régulièrement autorisées à fonctionner à nouveau depuis la Libération échappaient au transfert.

10 La SNEP attribuait à des entreprises de presse, en propriété ou location, les biens qui lui avaient été transférés et dévolus, suivant des plans de répartition établis pour Paris et le reste de la France. Une indemnisation était prévue pour les « actionnaires de bonne foi » et les anciens propriétaires qui n'avaient pas fait l'objet d'une condamnation pour faits de collaboration ou de commerce avec l'ennemi.

11 Les procédures de transfert étaient longues et complexes. L'attribution ne pouvait en outre intervenir avant le vote d'un nouveau statut de l'entreprise de presse : or les divers projets présentés n'aboutissaient pas. Cette impossibilité de l'attribution retardait d'autant les indemnisations des anciens propriétaires non condamnés et actionnaires « de bonne foi ». La SNEP "avait dû prendre en charge sans support financier des biens disparates, qui, détachés de leur contexte, avaient perdu une grande partie de leur valeur $»^{3}$. 
Une seconde loi relative à la presse, du 2 août 1954, allait permettre de débloquer la situation ${ }^{4}$. Toutes les décisions de transfert en vigueur à la date de publication de la loi étaient validées, et aucun nouveau transfert ne pourrait être opéré. Les entreprises de presse pouvaient acquérir des biens jusqu'alors gérés par la SNEP. Une «commission nationale de répartition des biens de presse » examinait et contrôlait les demandes. Les entreprises attributaires pouvaient conclure des contrats avec les anciens propriétaires ou leurs ayants droit. L'indemnisation des anciens propriétaires devenait possible, de même que celle des créanciers des entreprises transférées, notamment le personnel, grâce à la création d'un fonds spécial. La SNEP fut réorganisée en conséquence en décembre 1955.

\section{La collecte des fonds au lendemain de la Seconde Guerre mondiale et les premières entrées d'archives photographiques de presse aux Archives nationales}

La création de deux nouveaux services menant une politique active de prospection permit l'entrée d'archives de presse, par l'intermédiaire de la SNEP.

D'août à décembre 1944, les Archives nationales entreprirent une vaste opération de récupération des archives laissées à Paris par les services des administrations allemandes et à Vichy par l'État français. Pour faire face aux arrivées massives (près de quarante tonnes en l'espace d'une dizaine d'années), et réactiver la collecte des archives des ministères en activité, est instituée en 1947 une "section contemporaine ", employant une dizaine de personnes.

Parallèlement aux efforts déployés pour collecter les fonds publics, Charles Braibant, directeur des Archives de France et des Archives nationales, fit en sorte de favoriser la sauvegarde de fonds d'origine privée. En 1949, il diffusa une enquête auprès des services d'archives départementaux, destinée à répertorier les fonds d'archives économiques et privées existant dans le ressort des départements. Il instaura aux Archives nationales un «service du Microfilm, des Archives économiques et des Archives privées " ${ }^{5}$ pour la conservation de ce type de fonds, trop souvent négligés ou détruits, tandis que le microfilmage permettait d'assurer un "archivage de sécurité " ou la copie pour la communication. La même année, il constitua un comité pour la sauvegarde des archives privées, afin d'attirer l'attention des propriétaires sur la valeur historique de ces documents et de susciter leurs dons et dépôts dans les services d'archives.

Charles Braibant déclarait se préoccuper du sort des archives relevant de la SNEP ${ }^{6}$. En 1953, Jacqueline Piatier, responsable du service de documentation du Monde, proposa de céder des dossiers documentaires du journal Le Temps, conservés dans des locaux du Monde qui souhaitait s'en défaire : ce fut le point de départ des négociations concernant les archives des anciens journaux séquestrés. Les Archives nationales, intéressées par ces articles parus dans Le Temps, classés par matières et dont les plus anciens remontaient à 1864, se mirent aussi en relation avec la SNEP, qui détenait les archives comptables du Temps.

17 Michel François, responsable du Service des archives économiques et privées, était sur le point d'obtenir de la SNEP le versement des archives du Temps, et aussi celles du Matin, du 
Journal des débats, du Petit Parisien et de L'Euvre, mais l'opération échoua ${ }^{7}$. La SNEP connaissait, à un an de la promulgation de la seconde loi sur la presse, une situation de blocage dans la gestion des biens qui lui étaient confiés, et se dérobait à tout accord concernant la remise d'archives.

Par ailleurs, Marie-Thérèse Chabord, conservateur à la Section contemporaine, apprenait, au cours de l'été 1953, que des archives photographiques étaient entreposées dans un local dépendant de la SNEP, au 127 avenue des Champs-Élysées. Sur place, « au milieu de vieux bottins et de Journaux officiels", se trouvaient des tirages en quantité assez importante, ainsi que des négatifs sur plaques de verre 8 . C'est ainsi que fut acté en octobre 1953 le versement de "six caisses de photographies provenant des entreprises dévolues", ainsi que de " 4 tonnes de plaques de photographies d'agences photographiques de presse (principalement Trampus et Fama, 1940-1944)». Les documents furent évacués pour faire place nette ${ }^{9}$, tandis que des livres, brochures et autres papiers conservés dans le même lieu furent pilonnés, remis à la bibliothèque de la SNEP ou à d'autres établissements. Cette première entrée d'archives photographiques de presse s'apparentait à un sauvetage, alors que la SNEP tergiversait quant au versement des archives de fonctionnement des organes de presse.

\section{La politique de collecte des fonds de presse : le cas des archives photographiques}

19 À partir de 1956, la direction des Archives de France instaura une politique concertée de collecte des archives de presse au niveau national, sous l'égide du secrétariat d'État aux Arts et Lettres et avec la collaboration de l'Institut français de presse. Ces mesures concernaient d'abord les archives administratives et comptables des entreprises de presse, mais prenait aussi en compte le sort des archives photographique des journaux et agences, ainsi que leur orientation vers la Bibliothèque nationale ou les Archives nationales.

20 Au début du mois de mars 1956, Edmond Sidet, directeur de cabinet du secrétaire d'État aux Arts et Lettres, alerta Charles Braibant sur les possibles dispersion et destruction des archives de la SNEP, qui devait en principe cesser ses activités, et lui recommanda de contacter Fernand Terrou, chef du service juridique du secrétariat d'État à l'Information ${ }^{10}$ et membre du conseil d'administration de la SNEP.

21 À la suite de cette sollicitation, Charles Braibant constitua une commission de travail ${ }^{11}$ sur les archives de presse, qui réunissait plusieurs personnalités, dont le président-directeur général de la SNEP, Jean Mottin, Fernand Terrou ainsi que Claude Bellanger, directeur du Parisien libéré.

22 Peu auparavant, dans une note qu'il adressait au directeur des Archives de France ${ }^{12}$, Edmond Sidet précisait : « ...un de mes amis ${ }^{13}$ me fait observer l'intérêt qui s'attacherait à ce que la documentation photographique ${ }^{14}$ des journaux condamnés et non amnistiés soit également transférée, si possible à la Bibliothèque nationale (au département des Périodiques).»

23 La question des photographies allait être brièvement évoquée par Fernand Terrou ${ }^{15}$ lors de la séance de la commission. À propos des biens des journaux et en particulier de leurs archives, attribués à de nouvelles entreprises de presse, il signalait que ces dernières devaient autoriser leur remise aux Archives nationales, et qu'il y aurait une distinction à 
faire entre «les archives qui peuvent être utilisables, c'est-à-dire les photographies, coupures, etc. et les archives administratives qui ne peuvent leur être d'aucune utilité ». Les journaux pouvaient toujours utiliser ces ensembles iconographiques pour illustrer des articles relatifs à des personnalités, ou à des faits relativement récents. Les photographies, en raison de cet usage, ne semblaient pas une catégorie de documents susceptible d'entrer rapidement et en masse dans les services d'archives.

Ceci réglait la question des biens attribués à de nouvelles entreprises de presse. Quant à la "documentation photographique» appartenant aux journaux condamnés et non amnistiés, dont les archives relevaient de la SNEP, Charles Braibant ajoutait qu'elle se réduisait, d'après les premiers renseignements qu'il possédait, à peu de chose, et «la distraire des archives versées paraitrait contraire au principe du respect des fonds, règle fondamentale des Archives ${ }^{16}$ \%. Il soulignait le caractère indissociable de ces supports photographiques d'avec les archives administratives et comptables. On n'envisageait donc pas d'entrée de fonds photographiques de presse conséquents à cette période, exception faite de ce qu'avait déjà versé la SNEP en 1953.

La commission réunie par Charles Braibant avait pris plusieurs décisions destinées à favoriser la sauvegarde d'archives de presse. Premièrement, l'examen des fonds d'archives de presse encore conservés par la $\operatorname{SNEP}^{17}$ : à la suite de la visite de conservateurs du Service des archives économiques et privées fut versé ce qui restait des archives administratives du Matin, de L'Euvre et des Nouveaux Temps. Des dossiers provenant de journaux régionaux furent transférés aux archives départementales de l'Aisne, des Alpes-Maritimes, de l'Aube, du Maine-et-Loire, du Pas-de-Calais, de Seine-etOise et des Vosges.

La commission avait également adressé, élaborées par la direction des Archives de France et le secrétariat d'État à l'Information, des lettres circulaires aux présidents des douze entreprises de presse parisiennes graciées ou ayant acquitté une amende, pour leur suggérer de verser leurs archives inutiles, mais sans résultat.

Les mêmes lettres-circulaires furent adressées aux administrateurs des 46 sociétés de presse régionale et aux 33 directeurs d'archives des départements correspondants. Sur 20 réponses ( 11 de directeurs d'archives et 8 d'administrateurs de sociétés), une seule positive informait du versement aux archives de l'Hérault des archives du Petit Méridional.

Outre l'envoi des circulaires, une note intitulée «le versement d'archives privées aux Archives nationales ${ }^{18}$ " paraissait en mai 1956 dans l'organisme officiel de la Fédération nationale de la presse française pour attirer l'attention des directeurs et administrateurs de journaux sur les possibilités offertes par l'existence aux Archives nationales d'une Section des archives économiques et privées. À la suite de cette diffusion, les Archives nationales purent recueillir quelques dossiers du Matin, complétant les archives déjà reçues, ainsi que des archives de journaux régionaux, notamment La Sarthe. Quant aux archives photographiques du Matin, entreposées dans les locaux du journal boulevard Poissonnière à Paris, elles passeront à L'Humanité qui louera ces mêmes locaux en 1956 par l'intermédiaire de la SNEP'19. 


\section{La collaboration avec l'Institut français de presse (IFP) et la Bibliothèque nationale}

Les Archives nationales poursuivirent leur politique de collecte de fonds de presse, épaulées par l'Institut français de presse. Créé en 1937, l'Institut des sciences de la presse devint en 1951 l'Institut français de presse (IFP), et fut constitué en institut de l'Université de Paris en février 1957. Fernand Terrou, qui enseignait alors à l'Institut d'études politiques, en fut nommé directeur. L'IFP comprenait une section de méthodologie et de documentation, dont la première séance se tint en janvier $1958^{20}$. Présidée par Jean-Louis Crémieux-Brilhac, directeur adjoint de la Documentation française, elle réunit autour de Jacques Kayser, directeur adjoint de l'IFP, Charles Braibant, Jacqueline Mady, conservateur à la Section contemporaine des Archives nationales, Alice Gorgeon, responsable du centre de documentation de la Documentation française, Jean Prinet, conservateur en chef du département des Périodiques de la Bibliothèque nationale, Henri Michel, secrétaire général du Comité d'histoire de la Deuxième Guerre mondiale, et un représentant de la SNEP.

30

La séance était consacrée au problème des archives des journaux : archives saisies après la Libération, archives futures de la presse existante. Les échanges portèrent sur «les moyens les plus efficaces d'intéresser les journaux à leurs archives, et de coordonner les efforts des institutions pour recenser les fonds de presse ». Alors qu'on faisait état des actions déjà entreprises par les Archives (les lettres-circulaires envoyées aux entreprises de presse) et par l'IFP (établissement de listes de journaux confié à des étudiants), Jean Prinet insista sur une nécessaire coordination et fit remarquer qu'à la Bibliothèque nationale, «dont le rôle est de conserver non seulement les journaux mais la documentation qui les concerne», la recherche d'archives restait fragmentaire. Préoccupé depuis 1954 par le sort des archives photographiques des journaux, il avait constaté que des fonds très importants échappaient aux chercheurs parce qu'aucun organisme n'était qualifié pour les recevoir, qu'il s'agisse de négatifs sur verre, sur film ou de microfilms.

31 Les Archives nationales et la Bibliothèque nationale s'accordèrent en 1963 sur la répartition des éléments constitutifs des fonds de presse entre les deux institutions : les coupures de presse et les exemplaires de journaux à la première, et les archives proprement dites (administratives, contentieuses et comptables) aux secondes ${ }^{21}$. Le cas des archives photographiques n'était pas évoqué, et la question de l'attribution de ce type de fonds n'était pas tranchée. Près de vingt ans plus tard, la Bibliothèque nationale allait ainsi récupérer les archives photographiques du Journal, dont les archives administratives étaient entrées aux Archives nationales.

\section{La politique de collecte dans les années 1960-1970: les archives du Journal}

En 1965, l'IFP, alerté par le département des Périodiques de la Bibliothèque nationale, signalait aux Archives nationales l'existence des archives du Journal. Ce quotidien fondé en 1892 se replia à Lyon durant la Seconde Guerre mondiale et parut jusqu'à la 
Libération : alors suspendu, il cessa de paraître en juin $1944^{22}$. Très volumineuses, non classées et entassées dans un local appartenant au président du conseil de liquidation de la société anonyme Le Journal, les archives administratives du quotidien seront finalement sauvées et déposées aux Archives nationales en 1970.

Quant à ses archives photographiques, elles furent attribuées à L'Aurore après la Libération. En 1980, l'absorption de L'Aurore par Le Figaro étant imminente, le conservateur des Archives nationales qui avait suivi le dossier du Journal en 1970 examina ce fonds de près de 10000 cartons, auxquels s'ajoutaient les 5000 de la documentation photographique de L'Aurore. Il s'agissait de tirages sur papier, fournis par les agences de presse, légendés et classés thématiquement (personnalités, faits divers, etc.). Cependant, il n'existait ni répertoire ni fichier, et un certain désordre régnait, attribué à un déménagement ${ }^{23}$.

Bien que ce fonds pût compléter les archives du Journal, sa masse et «la modicité des moyens » des Archives de France (il s'agissait d'un achat) constituaient un frein, d'autant plus que des contacts venaient d'être pris avec L'Aurore par le responsable du département des Estampes et Photographies de la Bibliothèque nationale, en vue d'un complément éventuel de son dépôt légal. Aussi le directeur général des Archives de France, Jean Favier, prit-il la décision de laisser le fonds à la Bibliothèque nationale.

\section{Des objets problématiques : les négatifs sur verre}

Les archives du Journal renfermaient un petit ensemble de photographies de presse qui n'avait pas été compris dans le fonds photographique attribué à L'Aurore. Jusqu'à la fin des années 1980, il constitua le seul noyau photographique conservé dans les neuf fonds de presse répertoriés aux Archives nationales, avec les négatifs sur verre et les tirages provenant de la SNEP.

Reste que les quatre tonnes de plaques de verre reçues en octobre 1953 posaient problème en raison de leur support matériel, fragile et encombrant. Les Archives nationales les proposèrent au département des Estampes et Photographies de la Bibliothèque nationale, qui fit répondre "que la Bibliothèque nationale, n'étant pas organisée pour la conservation des plaques photographiques, ne gard[ait] que des collections de tirages $»^{24}$.

La Bibliothèque nationale indiquait cependant "la création prochaine, sous l'égide du Conseil international de la photographie ${ }^{25}$, d'un organisme chargé de la conservation des clichés ». La solution d'un versement dans une structure spécialisée dans la conservation de ce type de supports apparaissait la solution pour les Archives nationales, « qui ne sont pas plus outillées que la Bibliothèque nationale pour les conserver indéfiniment ».

On procéda à l'élimination des clichés altérés ou brisés, les boîtes de négatifs furent mises en cartons. Ces derniers, entreposés dans un des sous-sols de l'hôtel de Soubise, pesaient entre 10 et 15 kilos. Aucun inventaire des clichés n'ayant été remis au moment du versement, il aurait fallu exécuter des tirages sur papier pour tenter d'identifier l'objet des prises de vues; or les Archives nationales ne disposaient pas des moyens nécessaires pour une telle entreprise. Aucun instrument de recherche ne fut élaboré dans ces conditions, et le fonds est demeuré jusqu'à aujourd'hui inaccessible aux chercheurs ${ }^{26}$, alors que les tirages versés en 1953, en même temps que ces plaques de verre, ont pu être sommairement inventoriés ${ }^{27}$. 


\section{Dernières entrées de fonds photographiques de presse : Le Petit Parisien et Le Parisien libéré}

Claude Bellanger, directeur général du Parisien libéré et vice-président de l'IFP, avait alerté en 1969 le directeur général des Archives de France, André Chamson, sur la nécessité de sauver les archives du Journal, qui représentaient à son avis, avec celles du Matin et du Petit Parisien, à peu près tout ce qui subsistait de la grande presse de la III $^{e}$ République $^{28}$.

Il n'est donc pas surprenant que les archives du Petit Parisien, propriété du Parisien libéré, aient fait l'objet dans les années 1960 d'un début de classement et d'un inventaire par un conservateur des Archives nationales, en liaison avec l'IFP ${ }^{29}$. De plus, la menace d'un transfert des archives dans une cave insalubre, à l'occasion d'un déménagement des services du Parisien libéré, plaidait pour un transfert du fonds aux Archives nationales. Celui-ci sera réalisé en janvier $1989^{30}$.

41 Les archives photographiques ${ }^{31}$ du Petit Parisien comportaient environ 30000 tirages sur papier et près de 10000 négatifs sur verre. Il s'agissait là d'un fonds numériquement important, mais moins massif que le fonds versé en octobre 1953 par la SNEP. On consacra les moyens nécessaires à son traitement matériel et à son inventaire, qui occupa deux personnes à temps partiel durant trois ans et demi, pour s'achever à la fin de l'année 1996 32 .

Le Parisien libéré, devenu Le Parisien et ayant déménagé à Saint-Ouen, déposa une partie de ses propres archives, constituées de 4500 boîtes métalliques contenant des dossiers produits dans les années 1950-1970, une partie des archives de son service photographique (tirages), ainsi que les archives du Petit Parisien, qui comprenaient les collections reliées du journal, les livres de comptabilité, les dossiers de presse et de photographies du quotidien ${ }^{33}$. Les exemplaires de journaux ne furent pas retenus, et un contrat de dépôt fut établi pour ces fonds à la fin de l'année $1990^{34}$.

L'arrivée de ces deux ensembles volumineux de photographies et les moyens mis en œuvre pour leur traitement s'inscrivent dans un contexte nouveau pour le milieu des archives, traditionnellement tourné vers l'écrit : la reconnaissance de la valeur historique de la photographie et de l'image en général. On peut citer à cet égard la journée d'étude du 9 décembre 1986 organisée par l'Association des archivistes français, consacrée à «la place des archives dans le monde de la communication $»^{35}$, au cours de laquelle l'historien Denis Maréchal présenta une communication sur l'usage par les historiens des collections de photographies de presse, issue de sa thèse soutenue la même année, sous la direction de Jean-Noël Jeanneney : La photographie, quelle source pour l'histoire?.

Avec l'entrée des archives du Petit Parisien et du Parisien libéré s'achève la période de prospection et de collecte inaugurée par Charles Braibant, à la faveur des bouleversements intervenus dans le monde de la presse à la Libération.

Le paysage administratif avait aussi évolué ${ }^{36}$ : en 1993 était inauguré à Roubaix le Centre des archives du monde du travail ${ }^{37}$, ce qui entraîna la disparition de la Section des archives d'entreprises des Archives nationales, elle-même issue de la scission en 1970 de la Section des archives économique et privées en deux services, d'une part la Section des archives personnelles et familiales, et d'autre part la Section des archives d'entreprises, de presse et d'associations. 
La collecte destinée à la série AR s'orienta désormais vers les archives personnelles de directeurs de journaux, de journalistes de la presse écrite et audiovisuelle, certains ayant aussi exercé des activités dans les domaines politique, syndical, littéraire et artistique. Ces fonds retracent des parcours individuels, et les photographies conservées s'apparentent à celles de tout autre fonds familial ou personnel: albums et tirages réalisés à l'occasion des activités officielles et professionnelles, souvenirs de famille.

\section{Traitement archivistique des fonds}

Le classement et la description des archives photographiques du Petit Parisien et du Parisien libéré ont respecté l'organisation originelle en dossiers thématiques. En revanche, le traitement des phototypes versés par la SNEP, en particulier les négatifs sur verre, nécessitera de reconstituer des sous-ensembles thématiques et chronologiques.

\section{Versement 19771612 : SNEP}

Plusieurs versements de la SNEP ont été rassemblés sous une première cotation, la soussérie 7 AR (SNEP) ${ }^{38}$. Le versement 19771612 regroupe, dans ses articles 25 à 127 (fig. 1, fig. 2), les tirages contenus dans les «six caisses de photographies provenant des entreprises dévolues », entrées aux Archives nationales en octobre 1953.

Figure 1

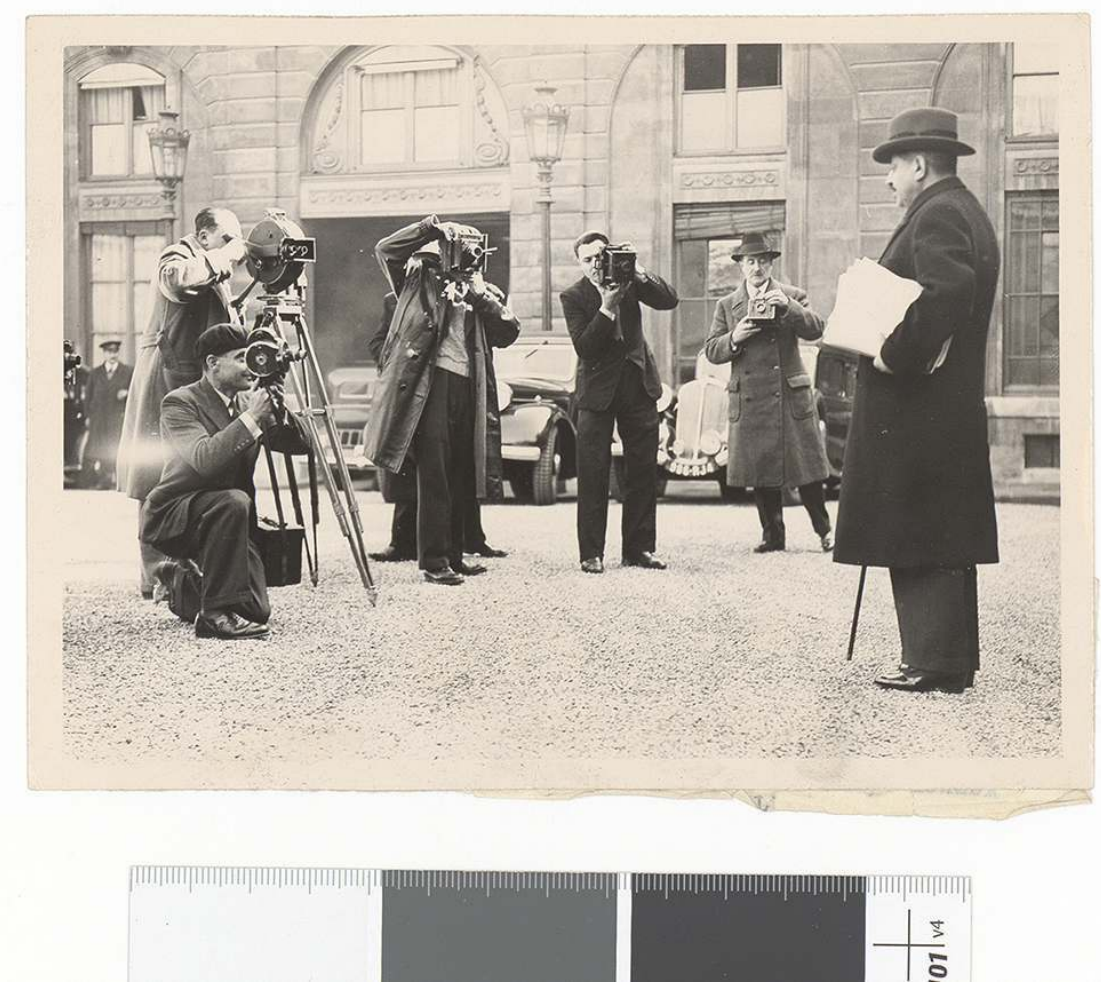

Sortie du conseil des ministres du 20 mars 1935 (recto). Arch. nat., 19771612/25.

Phot. Stéphane Méziache, Archives nationales, atelier photographique. (c) Agence Trampus. 


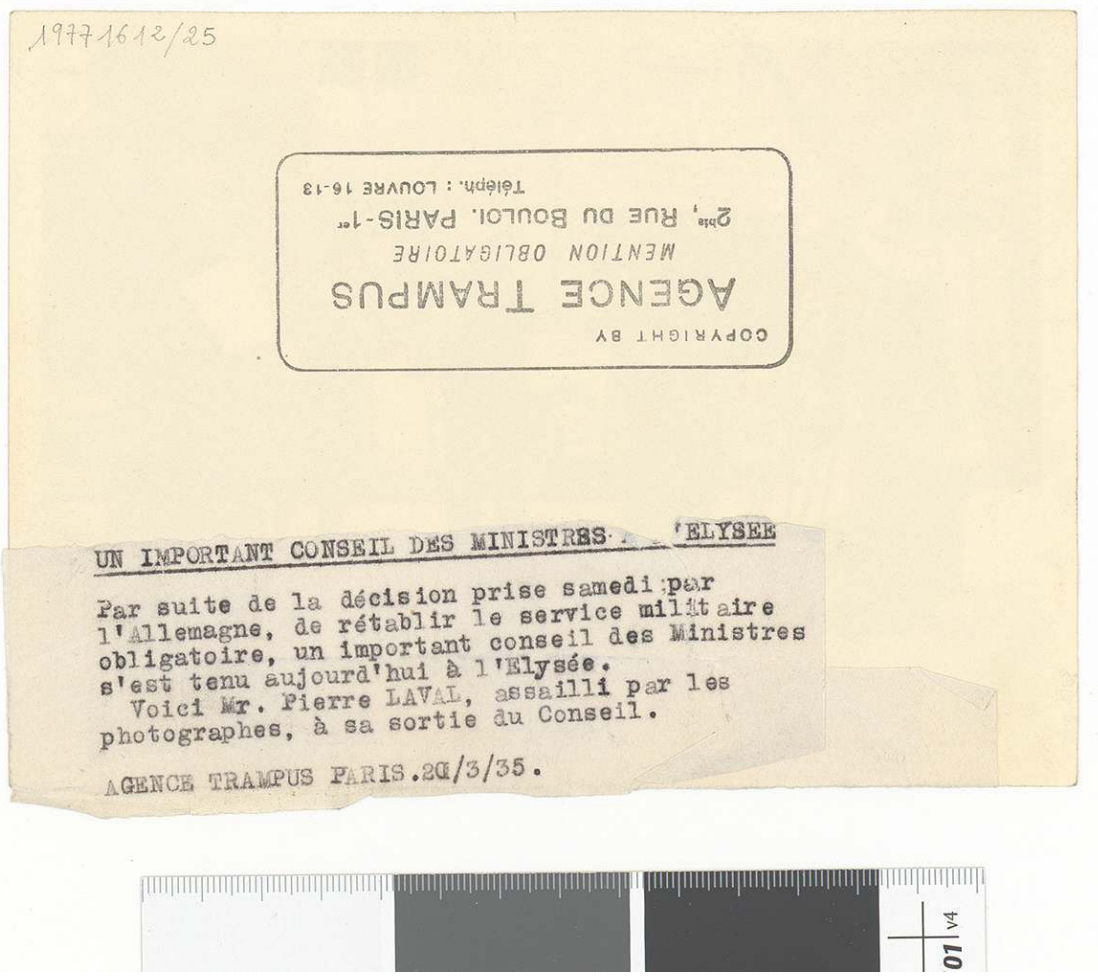

Sortie du conseil des ministres du 20 mars 1935 (verso). Arch. nat., 19771612/25.

Phot. Stéphane Méziache, Archives nationales, atelier photographique. ㄷ Agence Trampus.

Cet ensemble provient notamment des agences Trampus et Fama, de l'institut de propagande italien Luce, représenté en France par Trampus, et d'agences allemandes dont Trampus et Fama assuraient la diffusion ${ }^{39}$. Il faut signaler un ensemble d'albums de référence de l'agence Trampus, pour la période de juillet 1940 à juillet 1944 (fig. 3), comportant les numéros des tirages et de visa de censure (articles 29 à 49). Les numéros de tirages comportent le chiffre de l'année (40 à 44) suivi du numéro de reportage, puis du numéro d'ordre du cliché dans le reportage (exemple: 430517-2: $2^{\mathrm{e}}$ cliché du reportage 517 de l'année 1943). 


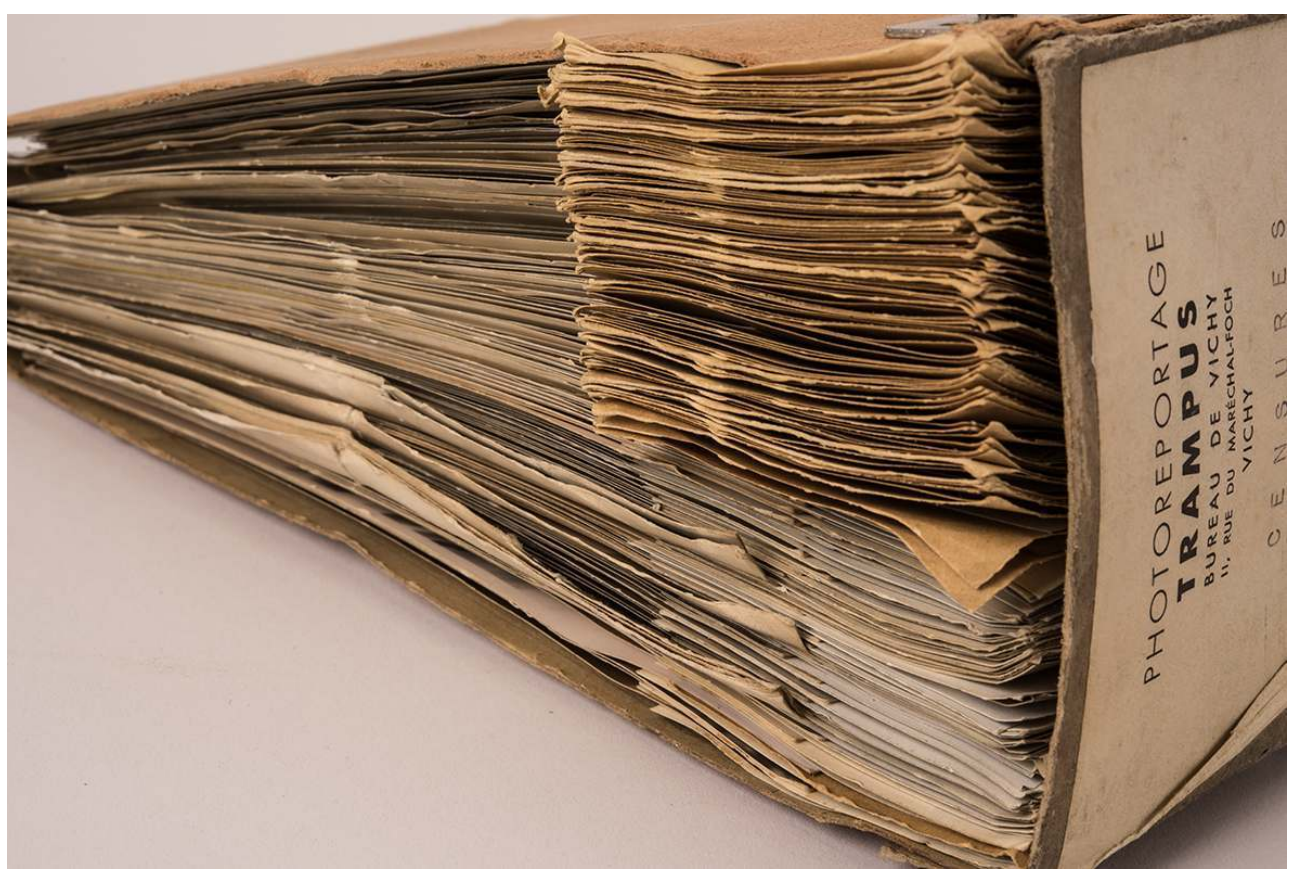

Album de référence de l'agence Trampus. Arch. nat., 19771612/29.

Phot. Stéphane Méziache, Archives nationales, atelier photographique. @ Archives nationales.

L'instrument de recherche du versement 19771612, très succinct, est actuellement disponible sous forme de répertoire numérique dactylographié. Le travail sur ce fonds consistera à reprendre le répertoire, en conservant les classements chronologique et par matière existants et en apportant des précisions sur les producteurs et utilisateurs des tirages à partir de leurs mentions dorsales et de leurs légendes. On rapprochera les numéros portés sur certains tirages et ceux qui sont inscrits sur les négatifs sur verre du versement 20130057.

\section{Versement 20130057 : SNEP}

51 Il s'agit des « quatre tonnes environ » de négatifs sur verre entrés aux Archives nationales en octobre 1953, en même temps que les tirages conservés dans le versement 19771612, et provenant, comme ce dernier, de Trampus, Fama, d'agences italiennes et allemandes.

Le nombre de négatifs est estimé entre 34000 à 50 000, conservés dans leurs 3416 boîtes d'origine. Cet ensemble comporte des reportages originaux, des reproductions de photographies et de documents iconographiques et cartographiques, des années 1930 jusqu'à la fin de la Seconde Guerre mondiale. Si les supports sont très majoritairement sur verre, on note également la présence de films nitrate.

Ce fonds fut d'abord enregistré sous le numéro 20020211. La cotation actuelle a été attribuée à l'occasion de l'emballage et de la mise en cartons des boites d'origine, en vue de leur déménagement du Centre des archives contemporaines de Fontainebleau au nouveau site des Archives nationales de Pierrefitte-sur-Seine.

Le classement de ce fonds s'articule en plusieurs étapes : 

nomenclatures inscrites sur les boîtes d'origine des négatifs (fig 4), avec vérification sommaire du contenu (conformité à la nomenclature externe, présence de négatifs nitrate). Les informations sont consignées sur un tableau faisant déjà apparaître des séries et ensembles spécifiques: reproductions, reportages, organisés en suites chronologiques ou simplement numériques.

Figure 4

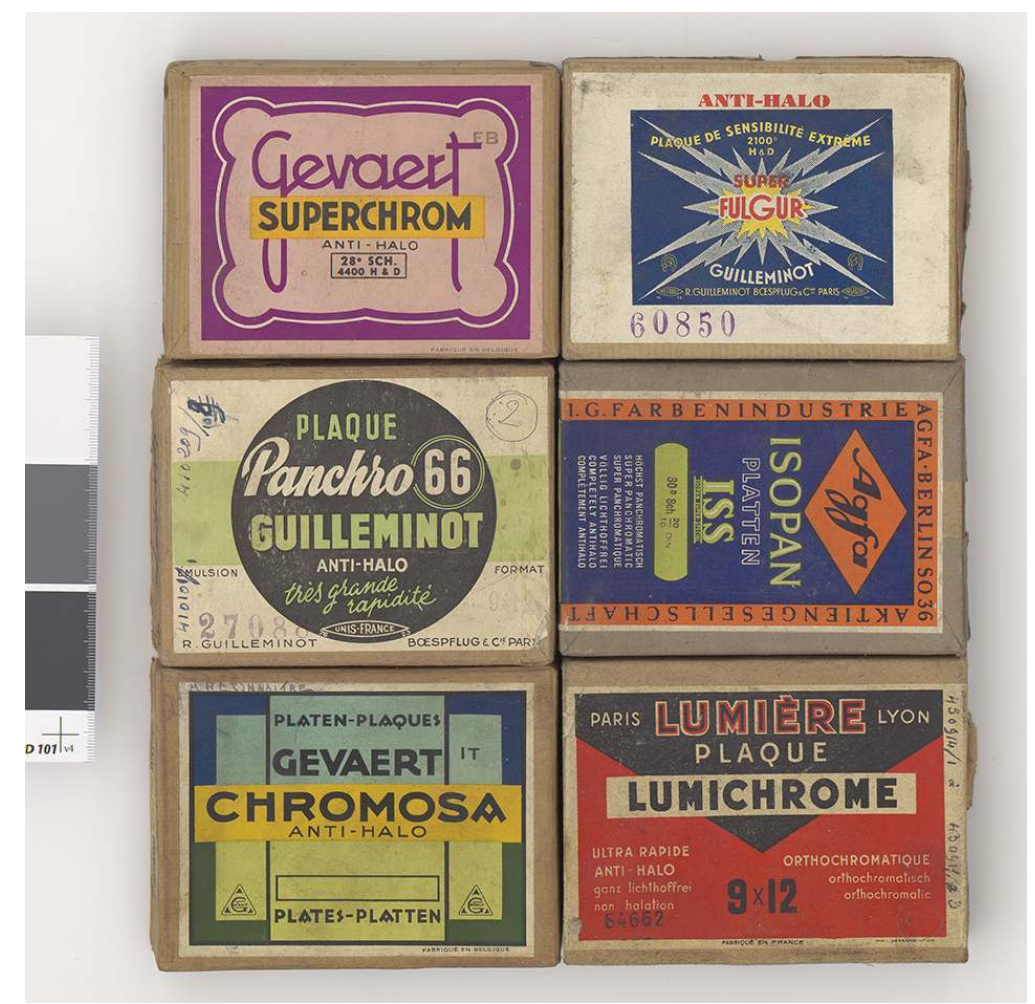

Boîtes originales de négatifs sur verre. Arch. nat., versement 20130057.

Phot. Stéphane Méziache, Archives nationales, atelier photographique. ㄷ Archives nationales.

2- le choix d'un sous-ensemble du fonds constituant une suite logique (chronologique et de provenance) à traiter en détail, cette fois-ci en relevant exhaustivement les numéros d'ordre des négatifs, les papillons et légendes contenus dans les boîtes.

On les comparera notamment avec les albums de référence de l'agence Trampus présents dans le versement 19771612.

Parallèlement, le nettoyage et la restauration des négatifs, ainsi que leur reconditionnement en boîtes de conservation, sera entrepris.

Le traitement de ce fonds est inscrit au programme scientifique, culturel et éducatif (PSCE) 2017-2020 des Archives nationales ${ }^{40}$. Il s'effectuera en lien avec celui de la soussérie Z 6, qui rassemble les archives de la cour de justice du département de la Seine instituée fin 1944, ayant compétence pour juger les auteurs d'actes commis entre le 16 juin 1940 et la Libération qui révèlent une intention de favoriser les entreprises de l'ennemi. Cette sous-série, en cours de classement, conserve notamment des photographies issues des agences Trampus et $\mathrm{Fama}^{41}$. 


\section{AR : Le Journal}

60

Ce fonds, composé essentiellement de dossiers de fonctionnement conserve néanmoins 9 cartons de photographies des années 1920 à 1930, organisées dans leur ordre chronologique originel (fig. 5). Légendés et portant le cachet du service des ventes du Journal, il s'agit de tirages destinés à être exposés dans les vitrines des points de vente du quotidien : kiosques, brasseries, hôtels (cotes 8 AR/531 en partie, 533 à 540). Les cotes 531 et 540 renferment également quelques tirages et albums dont l'origine n'est pas spécifiée. Un instrument de recherche décrit sommairement l'ensemble ${ }^{42}$.

Figure 5

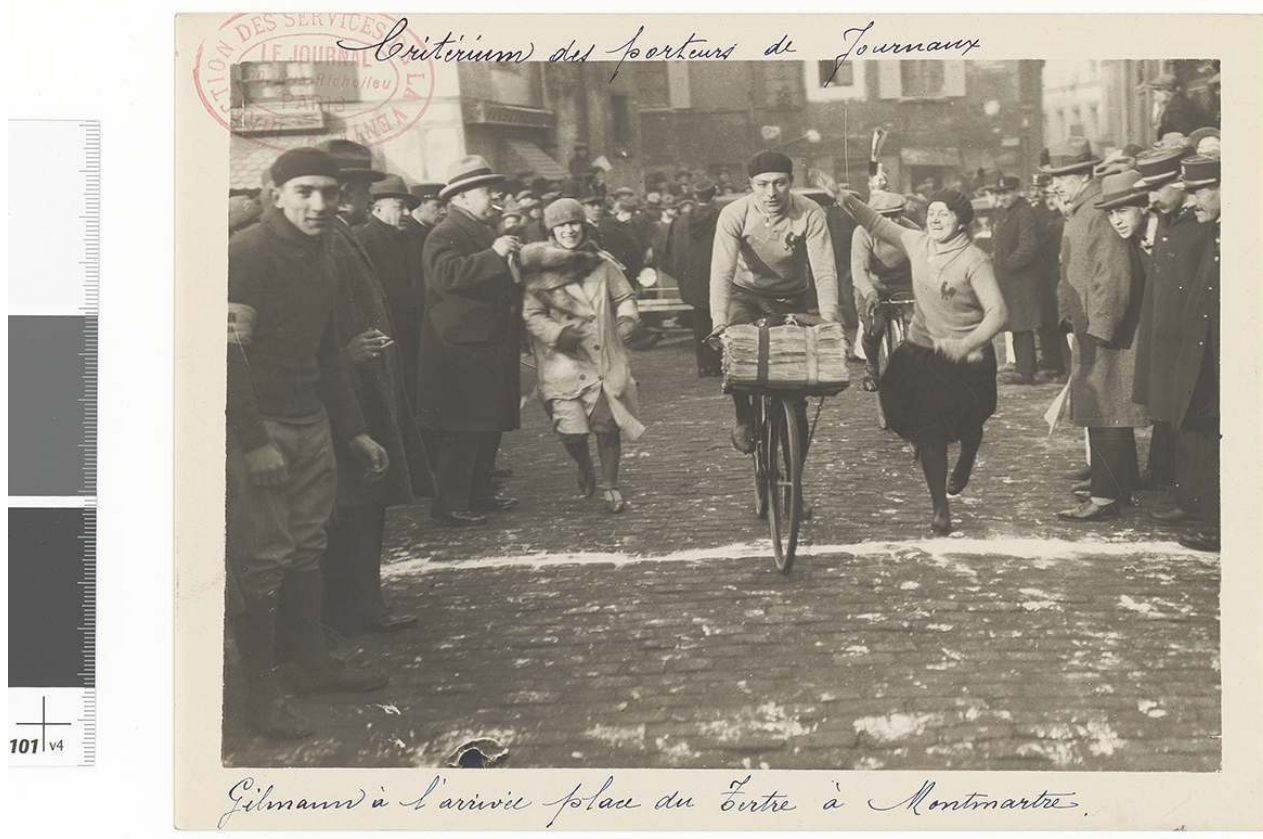

Criterium des porteurs de journaux [1928], Arch. nat., 8 AR/534.

Phot. Stéphane Méziache, Archives nationales, atelier photographique. () Service photographique du Journal.

\section{AR : Le Petit Parisien}

61 Le fonds se compose de registres de comptabilité, de dossiers biographiques (coupures de presse), d'archives relatives aux assemblées générales, et de supports photographiques (négatifs et tirages). Ces derniers proviennent à la fois du service photographique du journal (notamment les négatifs) et d'agences photographiques de presse. Elles comprennent :

62 - environ 32000 tirages sur papier, à savoir des photographies d'actualité, datées et légendées, allant des années 1930 à la Libération (fig. 6), classées dans leur ordre thématique d'origine (cotes $11 \mathrm{AR} / 757$ à 893). 
Figure 6

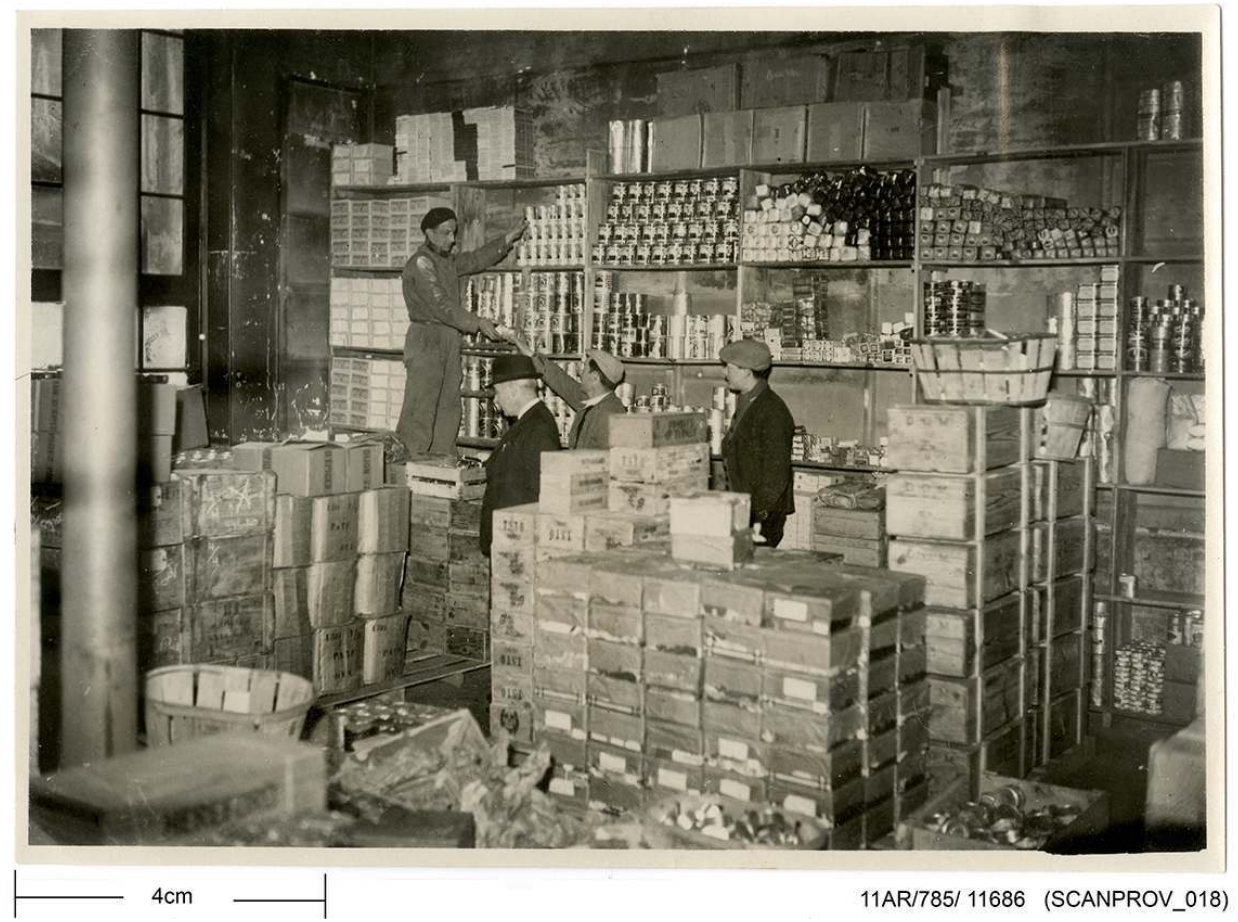

Vente de stocks de marché noir saisis par la police, 2 avril 1941. Arch. nat., 11 AR/785, photographie $n$ ${ }^{\circ} 11686$.

Phot. Roland Lachartre, Archives nationales, atelier photographique. (c) Service photographique du Petit Parisien, cl. Létondel.

63 - environ 10000 négatifs sur verre de format 9 x $12 \mathrm{~cm}$ (11 AR/ 699 à 756/B). Ils ne sont pas légendés et portent inscrit un numéro d'ordre, ou bien le numéro qui pendant la guerre et l'Occupation correspondait au numéro de visa de censure. On a pu faire correspondre près de 3000 de ces négatifs avec des tirages du fonds. L'ensemble des négatifs a été reconditionné en pochettes de papier neutre à 4 rabats et placé dans des containers d'aluminium anodisé.

64 Un instrument de recherche décrit l'ensemble du fonds ${ }^{43}$. Un vidéodisque se rapportant à 28000 tirages a été réalisé dans les années 1990: ses données sont en cours de retraitement.

\section{AR : Le Parisien libéré}

Le fonds comporte 320 cartons de tirages sur papier (12 AR/ 3294 à 3613), représentant une partie des archives photographiques du quotidien. Il s'agit de tirages et planchescontacts provenant de son service photographique et d'agences photographiques de presse. Le classement thématique d'origine a été conservé dans l'instrument de recherche ${ }^{44}$. 


\section{Conclusion}

Les archives des organes de presse ont été préservées en tant que patrimoine industriel : or les journaux et périodiques ne sont pas des produits industriels comme les autres, de simples biens de consommation, puisqu'ils touchent à la formation de l'opinion publique et des représentations collectives. En sauvant les archives de presse, on a d'abord envisagé la production de l'écrit, même si, dès les années 1950, on prit en compte également la part iconographique sans la considérer comme accessoire.

Reste que c'est de manière quasi fortuite que des archives photographiques de presse sont entrées aux Archives nationales. Sans les transferts de biens intervenus après la Libération et la création de la SNEP, les milliers de négatifs et de tirages de l'agence Trampus n'auraient sans doute jamais rejoint une institution publique mais, destin plus courant, auraient été rachetés ou détruits après la cessation d'activité de l'entreprise.

On peut qualifier ce patrimoine d'«adventice» en raison de la nature atypique du producteur, des supports matériels et du contenu visuel, qui le plaçaient hors du champ traditionnel de collecte des Archives et freinaient son traitement archivistique. Mais un premier jalon était posé, et le dépôt des fonds du Petit Parisien et du Parisien libéré s'est inscrit dès lors dans une logique de conservation qui reconnaissait à la photographie son entière qualité patrimoniale. La poursuite du travail sur le fonds de la SNEP s'inscrit dans cette continuité. Ce chantier de longue haleine s'apparente, par son ampleur et sa méthodologie, à celui des archives photographiques du Matin, poursuivi au musée de la Résistance nationale. Nul doute qu'à son terme, il offrira aux historiens une source de premier ordre, dévoilant la fabrique d'images placées sous étroite surveillance.

\section{NOTES}

1. - FEBVRE, Lucien, SCHMIDT, Charles. «L'organisation rationnelle des entreprises : les archives privées et l'histoire » [document électronique]. Annales d'histoire économique et sociale, $2^{\mathrm{e}}$ année, $\mathrm{n}^{\circ}$ 5, 1930, p. 64-66. www.persee.fr/doc/ahess_0003-441x_1930_num_2_5_1158 [consulté le 12/02/2018].

2. - BELLANGER, Claude, GODECHOT, Jacques, GUIRAL, Pierre et TERROU, Fernand. Histoire générale de la presse française : de 1940 à 1958. Paris : Presses universitaires de France, 1969-1976, t. 4, De 1940 à 1958, 1975, p. 200-209.

3. - Ibid., p. 208.

4. - Ibid., p. 385.

5. - FRANÇOIS, Michel. «Le service du Microfilm, des Archives économiques et des Archives privées aux Archives nationales ». [document électronique] La Gazette des archives, $\mathrm{n}^{\circ}$ 6, 1949, p. 14-17, https://www.persee.fr/doc/gazar_0016-5522_1949_num_6_1_1307 [consulté le 12/02/2018].

6. - Arch. nat. AB/XLII/36, « dossier de M. Braibant ", rapport à Monsieur le secrétaire d'État aux Arts et lettres, 28 mars 1956. 
7. - Arch. nat. $A B / X L I I / 36$, « dossier de M. Braibant », « note sur l'enquête relative aux archives administratives de presse », s. d., et « note sur l'affaire SNEP », s. d.

8. - Arch. nat. $A B / X L I I / 36$, deux notes, s. d.

9. - Arch. nat. $A B / X L I I / 36$, note de Dechêne, du bureau des archives et de la documentation de la SNEP, au directeur de la SNEP.

10. - Arch. nat. AB/XLII/36, « dossier de M. Braibant », note du 9 mars 1956.

11. - La commission se réunit le 28 mars 1956. Arch. nat. AB/XLII/36, « dossier de M. Braibant», « note sur l'enquête relative aux archives administratives de presse », s. d.

12. - Arch. nat. AB/XLII/36, « dossier de M. Braibant », note d'E. Sidet du 24 mars 1956.

13. - Cette suggestion émanait peut-être de Jean Prinet, directeur du département des Périodiques.

14. - L'emploi du terme a son intérêt : ces photographies forment une documentation, c'est-à-dire un ensemble rassemblé volontairement, de provenances diverses, subdivisé thématiquement pour répondre aux besoins du journal en matière d'illustration. Mais on peut considérer que ces mêmes ensembles, constitués par les journaux dans le cadre de leurs activités, répondent à la définition du fonds d'archives, a fortiori lorsqu'il s'agit de la production de négatifs interne au journal.

15. - Arch. nat. $\mathrm{AB} / \mathrm{XLII} / 36$, « dossier de M. Braibant », compte rendu de la réunion du 28 mars 1956.

16. - Arch. nat. AB/XLII/36, « dossier de M. Braibant », rapport à M. Sidet, du 28 mars 1956.

17. - Au siège situé 3 rue des Pyramides et dans un local en dépendant, au 127 avenue des Champs-Élysées.

18. - Il s'agit là d'un abus de langage. Le terme «versement » s'est imposé au cours du XIX ${ }^{\mathrm{e}}$ siècle pour désigner l'opération administrative et matérielle de transfert d'archives, du service qui les a rassemblées au service compétent pour les recevoir. Il s'applique aux archives publiques, les archives d'origine privée entrant dans un service d'archives par voie de don, achat ou dépôt révocable. Voir : État sommaire des versements faits aux archives nationales par les ministères et les administrations qui en dépendent. Paris: Imprimerie nationale, 1924, t.1, fasc. 1, p. XVII, XXXII, LXXXVI.

19. - Sur l'histoire de ces archives photographiques, voir dans la présente livraison: DEMERSSEMAN, Agathe. "Traitement et description d'un fonds photographique de presse en musée : le fonds dit «du Matin» au musée de la Résistance nationale», In Situ [En ligne], 36|2018, mis en ligne le 15 octobre 2018, consulté le 18 octobre 2018. URL: http:// journals.openedition.org/insitu/17511. Et : CLESSE, Joël et COURBAN, Maxime. «Organiser les masses : le traitement des archives photographiques du journal L'Humanité », In Situ [En ligne], 36|2018, mis en ligne le 17 octobre 2018, consulté le 18 octobre 2018. URL: http:// journals.openedition.org/insitu/17878.

20. - Arch. nat., AB XLII/36, « dossier de M. Braibant ». Compte rendu de la réunion du 17 janvier 1958 de la section de méthodologie de l'IFP.

21. - Arch. nat., AB XLII/36, dossier « archives de presse ", correspondance entre André Chamson et Julien Cain, 21 décembre 1962-8 février 1963.

22. - LACOUSSE, Magali. Les Sources d'archives relatives aux journaux et aux journalistes dans les fonds d'Archives privées (séries $A B X I X, A P, A Q, A R, A S$ ), XVIII ${ }^{e}-X X^{e}$ siècles. [document électronique], s. d. $\mathrm{h}$ ttp://www.archivesnationales.culture.gouv.fr/chan/chan/AP-pdf/AP-thematique-journaux-etjournalistes.pdf [consulté le 14/03/2018].

23. - Arch. nat., département des Archives privées, dossier du fonds 8 AR. Note du 14 mars 1980 de Yves Metman, chef du département des Activités scientifiques, culturelles et techniques, au directeur général des Archives de France, Jean Favier. 
24. - Arch. nat., $\mathrm{AB} / \mathrm{XLII} / 36$, dossier « Archives de presse ; archives de journaux trouvés à la SNEP et envoyés dans les départements ", " Note adressée à M. Laborie [chef du service du personnel de la SNEP] le 13 juillet 1956 ».

25. - Il s'agit sans doute du Comité international de la photographie, créé en 1958.

26. - Arch. nat. AB/XLIVb/22. Archives de l'atelier de photographie des Archives nationales, dossier « Recherche Hassner », 1974.

27. - Actuellement conservés dans le versement 19771612.

28. - Arch. nat., département des Archives privées, dossier du fonds 8 AR. Lettre du 25 janvier 1969.

29. - Il s'agissait de Pierre Cézard, conservateur à la Section contemporaine. Son inventaire a été publié dans AMAURY, Francine. Histoire du plus grand quotidien de la III ${ }^{e}$ République : Le Petit Parisien, 1876-1944. Paris : Presses universitaires de France, 1972. Voir Arch. nat., département des Archives privées, dossier du fonds $11 \mathrm{AR}$. Note, [1988] et l'introduction du répertoire numérique dactylographié du fonds 11 AR par Armelle Daumas et Véronique Dignac, 1997.

30. - Arch. nat., AB/XXXIV/14, lettres de Jean Favier des 22 novembre 1988 et 3 décembre 1990.

31. - DAUMAS, Armelle. « Un projet de vidéodisque inscriptible : le fonds du «Petit Parisien » au service des archives d'entreprise ». [document électronique], La Gazette des archives, $\mathrm{n}^{\circ}$ 152-153, 1991, p.133-135. https://documentfr.com/the-philosophy-of-money.html?utm_source=unprojet-de-videodisque-inscriptible-le-fonds-du-petit-parisien-au-service-des-archives-dentreprises [consulté le 21/03/2018].

32. - Arch. nat., département des Archives privées, dossier du fonds 11 AR. " Note au lecteur ", décembre 1996.

33. - Arch. nat., $\mathrm{AB} / \mathrm{XXXIV/14}$, lettre de Jean Favier du 22 novembre 1988 à M. Hamon, du Parisien

34. - Arch. nat., AB/XXXIV/14, contrat de dépôt du 30 novembre 1990.

35. - La Gazette des archives, $\mathrm{n}^{\circ} 140,1988$. [document électronique], www.persee.fr/issue/ gazar_0016-5522_1988_num_140_1 [consulté le 21/03/2018].

36. - État sommaire des fonds d'archives d'entreprises (série AQ), de presse (série AR) et d'associations (série AS) conservés aux Archives nationales. Paris : Archives nationales, 2007, p. 10-13.

37. - Dénommé Archives nationales du monde du travail depuis 2006. Les fonds d'entreprises des Archives nationales, à quelques exceptions, y furent transférés. http:// www.archivesnationales.culture.gouv.fr/camt/ [consulté le 21/08/2018].

38. - Voir: https://www.siv.archives-nationales.culture.gouv.fr/siv/rechercheconsultation/ consultation/pog/consultationPogN3.action?pogId=FRAN_POG_05\&nopId=p-

aaj0chpfo--17arfk8qjly85\&search=7AR [consulté le 21/08/2018].

39. - Notamment sur l'histoire des agences Fama et Trampus, voir: DENOYELLE, Françoise. La Photographie d'actualité et de propagande sous le régime de Vichy. Paris: CNRS Éditions, 2003, p. 187-197 et 231-238.

40. - Voir: http://www.archives-nationales.culture.gouv.fr/documents/10157/11403/ Programmation-scientifique-PSCE-2017-2020.pdf/3c5f31e8-2162-4d2f-9ff4-262b977093e0 [consulté le 20/03/2018].

41. - L'intérêt du point de vue politique des archives administratives des organes de presse versées aux greffes avait été évoqué lors de la séance de la commission réunie par charles Braibant le 28 mars 1956: il semblait alors prématuré de les récupérer, mais comme le fit remarquer Jacqueline Mady, elles viendraient aux Archives avec les versements des cours de justice.

42. - Voir: https://www.siv.archives-nationales.culture.gouv.fr/siv/rechercheconsultation/ consultation/ir/consultationIR.action?

irId=FRAN_IR_004452\&details=false\&gotoArchivesNums=false\&udId=root\&auSeinIR=true

[consulté le 30/03/2018]. 
43. - Voir: https://www.siv.archives-nationales.culture.gouv.fr/siv/rechercheconsultation/ consultation/ir/consultationIR.action?

irId=FRAN_IR_004455\&details=false\&gotoArchivesNums=false\&udId=root\&auSeinIR=true

[consulté le 30/03/2018].

44. - Voir: https://www.siv.archives-nationales.culture.gouv.fr/siv/rechercheconsultation/ consultation/pog/consultationPogN3.action?nopId=p-

aaj0cprsk--1kc99ro3uhf18\&pogId=FRAN_POG_05\&search=[consulté le 30/03/2018].

\section{RÉSUMÉS}

Les Archives nationales conservent cinq ensembles de photographies de presse issus d'agences et de journaux. L'entrée de ces fonds résulte de la politique de sauvegarde des fonds de presse initiée à partir des années 1950 conjointement par les Archives nationales, la Bibliothèque nationale et l'Institut français de presse, ainsi que des versements effectués par la SNEP (Société nationale des entreprises de presse), organisme public chargé de la gestion des biens de presse confisqués à la Libération. Si les archives de fonctionnement des journaux ont été collectées de manière privilégiée en tant que sources historiques de premier plan, les fonds photographiques constitués par les organes de presse ont également été pris en compte; toutefois leurs caractéristiques matérielles, (notamment en ce qui concerne les négatifs sur verre) ont constitué dans un premier temps un frein à leur conservation et à l'élaboration d'instruments de recherche. Cette mise en perspective historique permet de mieux comprendre le traitement archivistique appliqué à ces ensembles photographiques collectés depuis les années 1950 jusqu'à la fin des années 1980, provenant du Journal, du Petit Parisien, du Parisien Libéré, et à deux versements de la SNEP. Les Archives nationales s'engagent aujourd'hui dans l'inventaire d'un fonds provenant essentiellement de l'agence Trampus, une des agences dont les biens ont été confiés à la SNEP. L'article expose la méthodologie spécifique appliquée à ce type de supports.

The French National Archives today holds five collections of press photographs coming from agencies and newspapers. These collections arrived at the Archives as a result of a new policy of safeguarding press archives, initiated from the 1950s by the French National Archives, the National Library and the French Press Institute. Other papers came from the SNEP, the national society of press enterprises which was a public body responsible for the management of press properties confiscated in the period immediately after the Second World War. This historical perspective helps us understand the archival treatment applied to each collection. The National Archives is now engaged in the inventory of a collection coming primarily from the Trampus agency, one of the agencies whose property was acquired by the SNEP. The article discusses the specific methodology applied to these types of documents. 


\section{INDEX}

Keywords : archives, archival collections, departemental archives, National Archives, private archives, public archives, Bibliothèque nationale, Bellanger (Claude), Braibant (Charles), archival collecting, Favier (Jean), Institut français de presse (IFP), research instruments, newspaper, Le Petit Parisien, Le Parisien libéré, photography, press, Prinet (Jean), Société nationale des entreprises de presse (SNEP), Terrou (Fernand), Trampus (agency)

Mots-clés : archives, archives (fonds d'), archives départementales, Archives nationales, archives privées, archives publiques, Bibliothèque nationale, Bellanger (Claude), Braibant (Charles), collecte, Favier (Jean), Institut français de presse (IFP), instrument de recherche, journal, Le Petit Parisien, Le Parisien libéré, photographie, presse, Prinet (Jean), Société nationale des entreprises de presse (SNEP), Terrou (Fernand), Trampus (agence)

\section{AUTEUR}

\section{SANDRINE BULA}

Responsable de la mission photographie, Archives nationales sandrine.bula@culture.gouv.fr 